\title{
Compact CPV - Sustainable Approach for Efficient Solar Energy Capture with Hybrid Concentrated Photovoltaic Thermal (CPVT) System and Hydrogen Production
}

\author{
Muhammad Burhan* \\ Water Desalination and Reuse Centre (WDRC), Biological and Environmental Science \& Engineering (BESE), King \\ Abdullah University of Science and Technology, Saudi Arabia \\ Muhammad Wakil Shahzad ${ }^{\dagger}$ \\ Water Desalination and Reuse Centre (WDRC), Biological and Environmental Science \& Engineering (BESE), King \\ Abdullah University of Science and Technology, Saudi Arabia \\ Kim Choon $\mathrm{Ng}$ \\ Water Desalination and Reuse Centre (WDRC), Biological and Environmental Science \& Engineering (BESE), King \\ Abdullah University of Science and Technology, Saudi Arabia
}

\begin{abstract}
Solar energy being intermittent in nature, can provide a sustainable, steady and high density energy source when converted into electrolytic hydrogen. However, in current photovoltaic market trend with $99 \%$ conventional single junction PV panels, this cannot be achieved efficiently and economically. The advent of the multi-junction solar cells (MJCs), with cell-efficiency exceeding 46\%, has yet to receive wide spread acceptance in the current PV market in form of concentrated photovoltaic (CPV) system, because of its system design complexity, limiting its application scope and customers. The objective of this paper is to develop a low cost compact CPV system that will not only eliminate its application and installation related restrictions but it is also introducing a highly efficient and sustainable photovoltaic system for common consumer, to convert intermittent sunlight into green hydrogen. The developed CPV system negates the common conviction by showing two times more power output than the flat plate PV, in tropical region. In addition, sunlight to hydrogen conversion efficiency of $18 \%$ is recorded for CPV, which is two times higher than alone electricity production efficiency of flat plate PV. As concentrated photovoltaic (CPV) system can operate at x1000 concentration ratio, therefore, such high concentration ratio requires heat dissipation from the cell area to maintain optimum temperature. With Such heat recovery, the hybrid CPVT system has shown solar energy conversion efficiency of $71 \%$.
\end{abstract}

\begin{abstract}
1. Introduction
The global warming circumstances of $1.5^{\circ} \mathrm{C}$ rise in the average ambient temperature [1-5] is deemed inevitable due to the excessive emission of green-house gases such as Carbon Dioxide [2]. Today, the renewable energy contributes only about $6.7 \%$ of the global energy demand [3]. The reason for the slow implementation of renewable energy sources can be attributed to their vulnerability to intermittency, low energy density and localized availability that makes these energy sources mediocre as compared to the conventional fossil fuel-based sources. Amongst all renewable sources, the solar energy has the highest potential [4] in meeting a significant portion of the total energy demand of future and for such an energy sources to achieve sustainability, its energy quality and supply continuity are to be improved [5].

Current photovoltaic(PV) systems for electricity generation, available hitherto, comprises the stationary single junction siliconbased solar cells such as mono-crystalline, poly-crystalline and thin films [6,7] with more than $98 \%$ share of photovoltaic market. Although such cells have a theoretical limit of $31 \%$ efficiency, the actual long term electricity rating is merely less than $8 \%$ [8]. The advent of the third generation multi-junction solar cells (MJCs), with the instantaneous cell-efficiency exceeding 46\% [9] (but the quantum limit is $86 \%$ ), has yet to receive wide spread acceptance in form of concentrated photovoltaic (CPV) system, in the current PV market because of its system design complexity in application. Conventional gigantic (big size) CPV systems require massive structures and open land spaces, limiting its application scope and customers. It is not simple to have compact CPV design as it demands more units of solar trackers or control hardware and they demand very high degree tracking accuracy as high as $0.1^{\circ}$. Therefore, if cost of the solar tracking units cannot be reduced then the compact CPV concept cannot compete with the conventional gigantic (big size) CPV design, however, without compromising on the required tracking accuracy.

On the other hand, despite high grade energy production as electricity, it is still intermittent and requires energy storage for steady power supply. While electrochemical storage in form of battery, does not make solar energy as viable option to replace fossil fuels [10]. Electrolytic production of hydrogen by splitting water using solar electricity not only provides a portable green energy but also acts as high density energy source, more than just energy storage $[11,12]$. The byproduct oxygen has many useful applications ranging from green disinfectant ozone to direct consumption in oxygen-based processes. Although, hydrogen production from solar energy has been reported to have very high efficiency i.e. recent literature of NERL reported that the
\end{abstract}

*Post-Doctoral Fellow, corresponding author, muhammad.burhan@kaust.edu.sa

${ }^{\dagger}$ Research Scientist

"Professor 
conversion of sunlight into hydrogen has achieved a "world record" of $16.2 \%$ via a photo-electrochemical (PEC) water-splitting process [13], surpassed the previous record of 14\% of Fraunhofer ISE and the California Institute of Technology [14,15]. However, to be a sustainable energy source, the production efficiency must be very high to meet the energy demand. The present study focuses on the utilization of the highly efficient CPV technology for the production of hydrogen as solar energy storage and sustainable future fuel. On the other hand, due to operation under high concentration, there is a lot of heat dissipation by MJC, which can be recovered for low temperature solar thermal applications.

We propose to have compact and low cost CPV system which can exploit the scale of mass production methods and can be placed on rooftop or open field applications. The proposed compact and low cost CPV design approach will eradicate the gigantic design complexities, with market boost by enabling it to tap one of the biggest potential of rooftop installation by common user, which current commercial CPV systems are not capable to do. Such proposed compact design will be utilized to develop a CPVHydrogen/Oxygen system to meet the sustainable future energy goals using solar energy. The produced hydrogen will be stored for ease, which acts as a portable energy to readily convert it back to electricity using fuel cell. Oxygen (O2) can be used to produce a disinfectant Ozone (O3) for its oxidation/cleaning potential. Such system will not only target domestic sector but majorly the industrial sector depending upon their use, by utilizing existing rooftop space with expected sunlight to electricity efficiency of $28 \%$, sunlight to hydrogen efficiency of $18 \%$ and sunlight to electricity/thermal efficiency of $71 \%$.

\section{Compact CPV Field Configuration}

In order to reduce overall hardware requirements of solar trackers, especially the electronic modules needed for conventional astronomical solar tracking, the position of the sun for initial guess of the solar tracker, will be obtained by solar geometry model [16-18] based upon the latitude, longitude, date and time of the tracker location. Azimuth and zenith are the two solar angles which are used to define the position of the sun, as given by equations (1) and (2).

If $\omega>0$,

If $\omega<0$,

$$
\theta_{a}=360-\left[90+\cos ^{-1} \frac{\sin \delta-\sin \left(90-\theta_{z}\right) \sin \phi}{\cos \left(90-\theta_{z}\right) \cos \phi}\right]
$$

$$
\theta_{a}=90+\cos ^{-1} \frac{\sin \delta-\sin \left(90-\theta_{z}\right) \sin \phi}{\cos \left(90-\theta_{z}\right) \cos \phi}
$$

and

$$
\theta_{z}=\cos ^{-1}\{\sin \phi \sin \delta+\cos \phi \cos \delta \cos \omega\}
$$

Where $\varnothing, \omega$ and $\delta$ are latitude, hour and declination angles respectively.

These solar position coordinates will be distributed to the entire CPV field of slave trackers with low cost wireless transceivers of Zigbee modules, Fig. 1. In this entire network, the central microcontroller will act as master which will send the coordinates of the position of the sun and the CPV trackers will act as slave to follow the received solar position as per their stage- 1 tracking to guess their initial position. Therefore, we can reduce the expensive electronic module requirements of the overall CPV field with reduced cost as only master microcontroller will be equipped with such units to act as a central information transmitter. For communication between Zigbee modules, following equations (3) and (4) are used to assign names to certain control box in CPV field, as explained in [19].

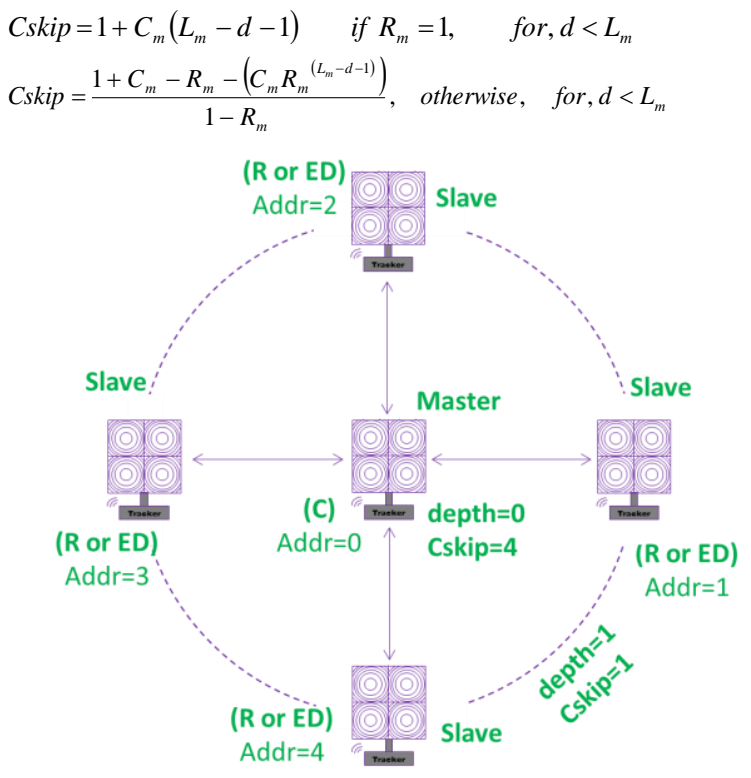

Fig. 1: Master-Slave Configuration for Compact CPV 


\section{Twin Lens Collimator based Solar Tracking Sensor}

In order to ensure the tracking accuracy required by the CPV module i.e. $0.1^{\circ}$, each tracker is equipped with solar feedback sensor, Fig. 2, which accounts for the fine adjustments of the individual tracker position in case of small difference in the solar position and the actual position of the tracker. In order to design a low cost but highly accurate and sensitive solar feedback sensor, an optical collimator with dual lens is used with an array of ordinary photo- sensors. In its simple form, the solar feedback sensor is designed to have a collimated bright spot at the center of the photo-sensor array. With the induced tracking error, the bright spot shifts from the center and if tracking error exceeds from the required tracking accuracy, the solar feedback sensor is aligned with the tracker such that the bright spot hits any of the photo-sensor in the array. The feedback sensor then gives the feedback signal as high, indicating tracking error and the tracker is adjusted such that the bright sport again comes in the center of the photo-sensor array. Another advantage of concentrated bright spot is to hit the photo-sensor with light intensity larger than their saturation limit and in such case, the sensor can only give extreme high or extreme low output. As a result we can use any ordinary photo-sensor in binary mode by avoiding its non-uniform response under the same light intensity.

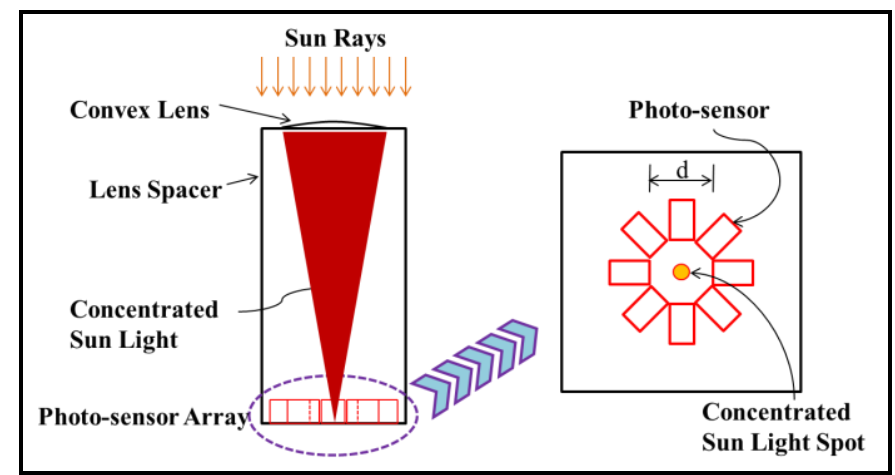

Fig. 2: Twin Lens Collimator based Solar Tracking Sensor

\section{Prototype of Compact CPV with Hydrogen system and Testing Methodology}

By adopting the novel and low cost solar tracking strategies, the prototype of compact CPV system is developed using triple junction InGaP/InGaAs/Ge solar cell and such a compact CPV system is then be used to build a pilot- setup of CPVHydrogen/Oxygen system to produce hydrogen and oxygen by electrolytic splitting of water through proton exchange membrane (PEM) electrolyzer, Fig. 3. The main reason to use PEM based electrolyzer is to have high purity of the gases without any extra energy intensive separation processes. The produced hydrogen and oxygen are compressed to be stored into portable cylinders for further use such as fuel cell or as disinfectant. Such developed system will be able to be installed anywhere either rooftop or open field, for domestic, commercial or industrial use, to make solar energy as sustainable energy source with steady power production. The performance of the CPV- Hydrogen/Oxygen system is analyzed in terms of conversion efficiency as the performance parameter for each component of the system are recorded through central data logging unit.
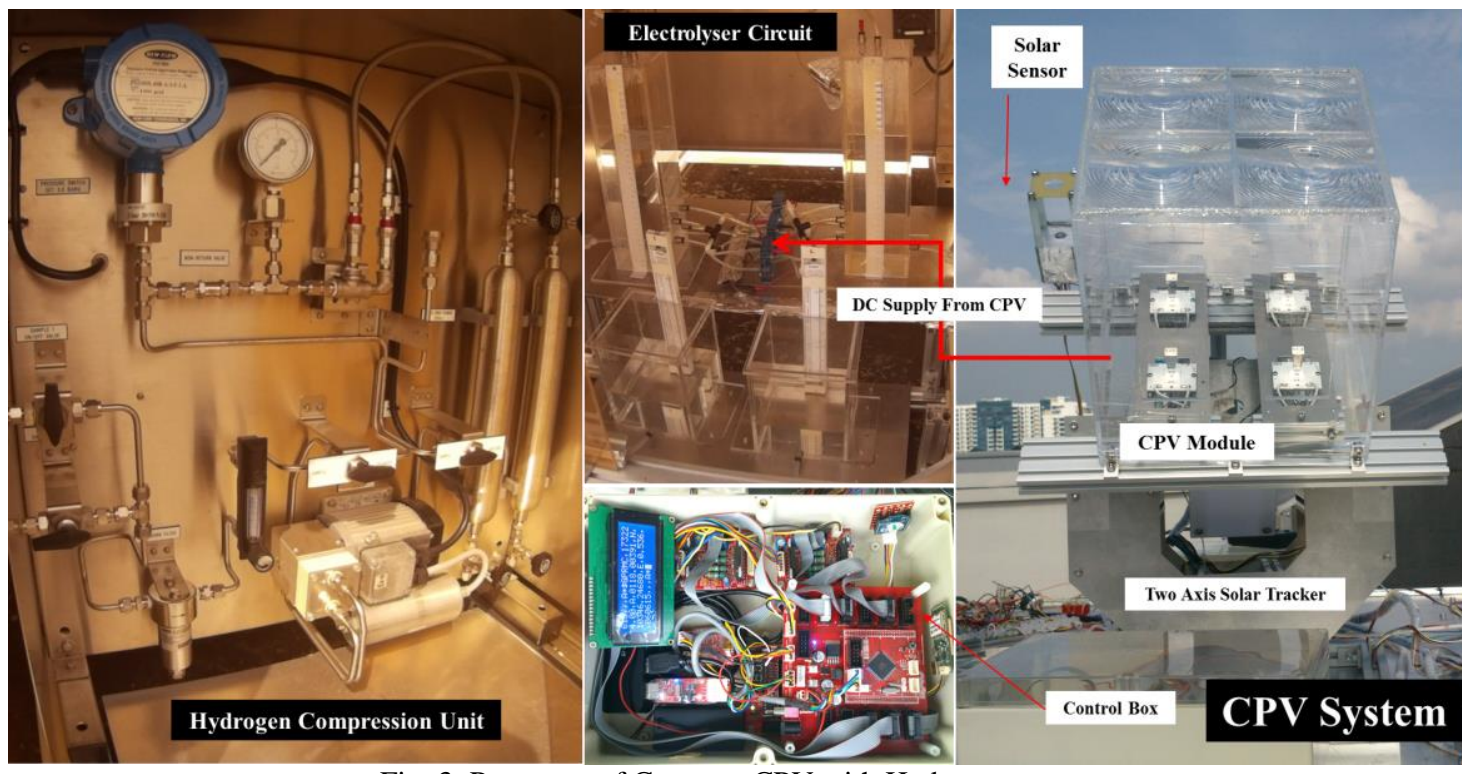

Fig. 3: Prototype of Compact CPV with Hydrogen system 
Based upon the energy received by the CPV system, overall sunlight to hydrogen efficiency can be calculated as

$$
\begin{gathered}
\eta_{E L}=\frac{\dot{n}_{E, H 2} 237200}{I_{E} \cdot U_{E}}=\frac{\eta_{E F} 1.23 N_{E C}}{U_{E}} \\
\eta_{S T H}=\frac{P_{\text {out }}}{P_{\text {in,solar }}} \frac{\left(\dot{n}_{E, H 2} 237200\right)}{\left(\frac{P_{C P V}}{\eta_{C P V}}\right)}=\frac{\eta_{E F} I_{E} 1.23 N_{E C}}{\left(\frac{P_{E}}{\eta_{C P V}}\right)}=\frac{\eta_{E F} I_{E} 1.23 N_{E C}}{\left(\frac{U_{E} I_{E}}{\eta_{C P V}}\right)}=\eta_{C P V} \frac{\eta_{E F} 1.23 N_{E C}}{U_{E}}
\end{gathered}
$$

The electrical power output of the CPV system is same as the electrical energy input of the electrolyser. The thermal power output of the CPV system is given by the equation (7).

$$
Q_{t h}=\left\{\left(I_{b} \times A_{c o n} \times \eta_{o p}\right)-P_{C P V}\right\} \times\left(1-Q_{\text {loss }}\right)
$$

Therefore, the efficiency of CPVT system can be calculated as:

$$
\eta_{C P V T}=\frac{P_{\text {out }-C P V T}}{P_{i n, \text { solar }}}=\frac{P_{C P V}+Q_{\text {th }}}{I_{b} \times A_{\text {con }}}
$$

In order to calculate the long term efficiency of the system in form of total energy output against total energy input, the average efficiency of the system can be calculated as [20]:

$$
\eta_{D N I}=\frac{\text { Electrical Rating }}{\text { Solar DNI Input }}=\frac{\left[\left(\sum_{i=1}^{t}\left(\frac{\left(V_{C P V} \cdot \mathrm{I}_{C P V}\right)_{i}-\left(V_{C P V} \cdot \mathrm{I}_{C P V}\right)_{i-1}}{2 \times A_{C}}\right)\right) \times \frac{365}{m}\right]}{\left[\left(\sum_{i=1}^{t}\left(\frac{\left(\mathrm{I}_{b}\right)_{i}-\left(\mathrm{I}_{b}\right)_{i-1}}{2}\right)\right) \times \frac{365}{m}\right]}
$$

\section{Results and Discussion}

The performance of developed compact CPV-Hydrogen/oxygen system is analyzed for whole day operation with received DNI (Direct Normal Irradiance), as shown in Fig. 4. Firstly, it can be seen that the system showed maximum CPV efficiency of $28 \%$, which is decreasing with decrease in DNI (Direct Normal Irradiance). The decrease in CPV efficiency is due to increase in the concentration at cell area, which cause increase in cell operating temperature. It can be seen that CPVT efficiency of $71 \%$ maximum is recorded, which is almost stable throughout the day. With increase in DNI (Direct Normal Irradiance), as CPV efficiency drops with increase in cell temperature, the thermal recovery increase. Therefore, the overall efficiency of CPVT system is almost steady throughout the day.

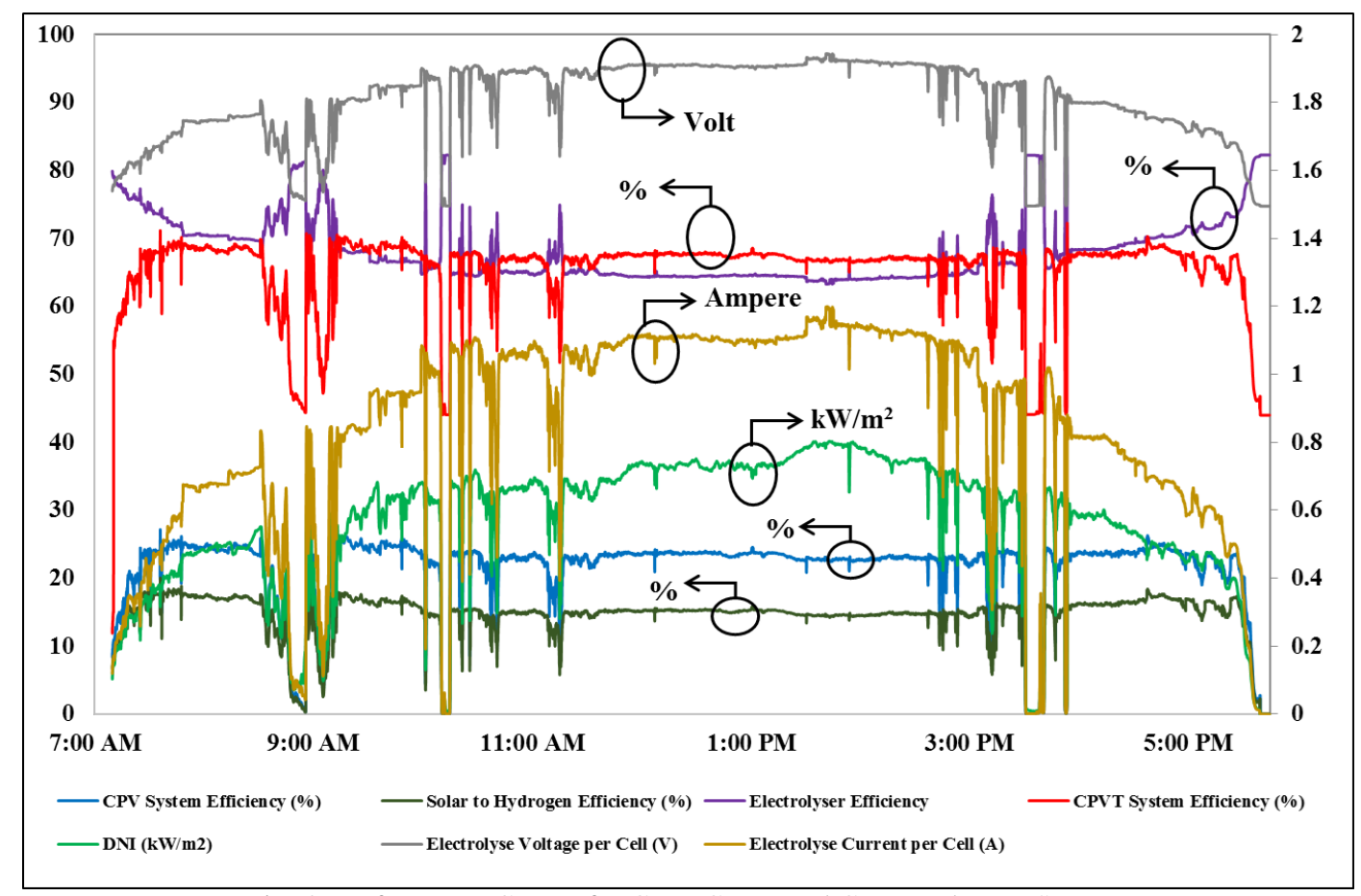

Fig. 4: Performance Curves for CPV, CPVT and CPV-Hydrogen System.

For CPV-Hydrogen/Oxygen, a maximum sunlight to hydrogen (STH) efficiency of $18 \%$ is recorded, which decrease with increase in temperature. It can be seen that the decrease in STH efficiency is due to decrease in the electrolyser efficiency. From 
equation (5), it is clear that the efficiency of electrolyser is inversely proportional to it operating voltage. As the CPV electricity is directly supplied to the electrolyser, therefore, with increase in DNI (Direct Normal Irradiance), the power output of CPV increases and so as the operating voltage of electrolyser. That is why, electrolyser efficiency is decreasing with increase in DNI (Direct Normal Irradiance).

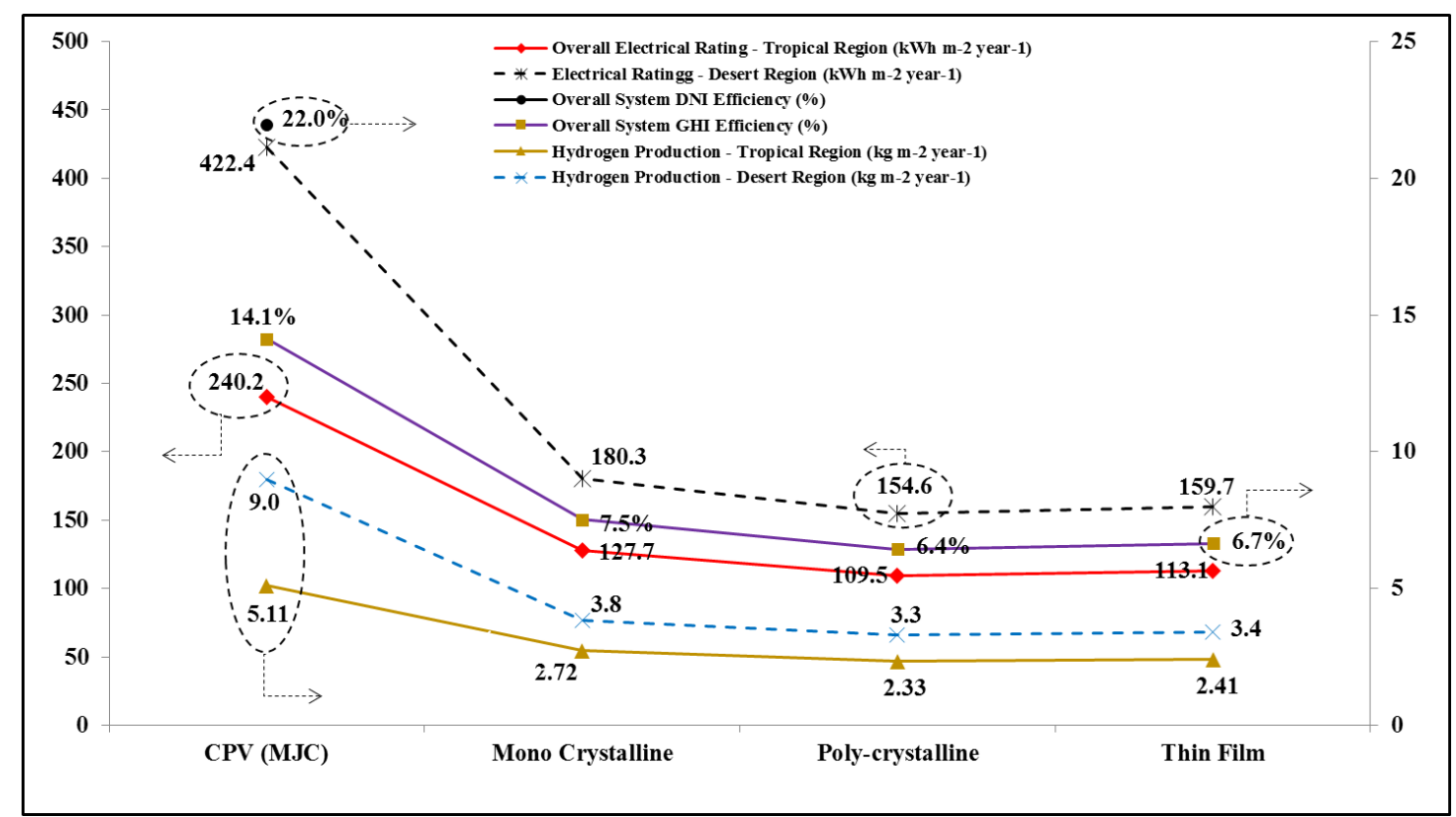

Fig. 5: Long Term Electrical Rating and performance of CPV and CPV-Hydrogen System

Figure 5 shows the long term field performance of CPV system and compares it with conventional PV systems installed in tropical weather condition of Singapore [21], in terms of its electricity and hydrogen production. The presented data shows has CPV has double the capacity of power production than conventional PV system, even in tropical weather conditions which are not considered suitable for CPV operation as it can only respond to beam radiations and tropical weather conditions have less share of beam radiations in received solar energy. In addition, it can also be seen that the CPV production boost to 3 time the conventional PV output in desert field condition, which is due larger share of beam radiations in the desert area. However, from hydrogen production point of view, CPV has hydrogen production potential of $5-9 \mathrm{~kg} / \mathrm{m}^{2} /$ year which is also $2-3$ times the potential of conventional PV. In current study, CPV-hydrogen system is presented as a future sustainable energy source with promising production potential and the CPVT system can achieve as high as $71 \%$ solar energy conversion efficiency, depending upon its application.

\section{Conclusion}

A design of compact CPV system is presented with in-house built solar tracker sensor and master-slave field configuration, to achieve overall less system cost without compromising the accuracy of the system. The system is designed to handle the installation related limitations of conventional gigantic (big size) CPV, which will boost the CPV installations in the rooftop of buildings like conventional PV. In addition, overall 71\% solar energy efficiency, $28 \%$ electrical efficiency and $18 \%$ hydrogen production efficiency shows huge performance potential of CPV than conventional PV. For CPVT application with $71 \%$ efficiency, although the whole energy cannot be utilized in high grade energy applications, but it can be properly utilized with effective heat recovery system applications. With such huge potential and the energy efficiency, concentrated photovoltaic (CPV) can provide a sustainable solar energy system for future green environment. In addition, such compact CPV system will boost the urban rooftop installation of CPV and so as its market share. This study also proves the feasibility and potential of CPV is the tropical weather conditions.

\section{References}

[1] Shafiei E, Davidsdottir B, Leaver J, Stefansson H, Asgeirsson EI. Comparative analysis of hydrogen, biofuels and electricity transitional pathways to sustainable transport in a renewable-based energy system. Energy 2015, 83, 614-627.

[2] Burhan M, Shahzad MW, Ng KC. Development of performance model and optimization strategy for standalone operation of CPV-hydrogen system utilizing multi-junction solar cell. International Journal of Hydrogen Energy 2017, 42(43), 26789-803.

[3] Burhan M, Chua KJE, Ng KC. Electrical rating of concentrated photovoltaic (CPV) systems: long-term performance analysis and comparison to conventional PV systems. International Journal of Technology 2016, 7(2), 189-196. DOI: 10.14716/ijtech.v7i2.2983. 
[4] Ng KC, Burhan M, Shahzad MW, Ismail AB. A Universal Isotherm Model to Capture Adsorption Uptake and Energy Distribution of Porous Heterogeneous Surface. Scientific Reports 2017, 7(1), 10634.

[5] Burhan M, Chua KJE, Ng KC. Sunlight to hydrogen conversion: Design optimization and energy management of concentrated photovoltaic (CPV-Hydrogen) system using micro genetic algorithm. Energy 2016, 99, 115-128.

[6] IPCC, Renewable Energy Sources and Climate Change Mitigation. Special Report of the Intergovernmental Panel on Climate Change. 2012.

[7] Burhan M., Chua KJE, Ng KC. Simulation and development of a multi-leg homogeniser concentrating assembly for concentrated photovoltaic (CPV) system with electrical rating analysis. Energy Conversion and Management 2016, 116, 58-71.

[8] Burhan M, Shahzad MW, Ng KC. Long-term performance potential of concentrated photovoltaic (CPV) systems. Energy Conversion and Management 2017, 148, 90-9.

[9] Burhan M, Oh SJ, Chua KJE, Ng KC. Double lens collimator solar feedback sensor and master slave configuration: Development of compact and low cost two axis solar tracking system for CPV applications. Solar Energy 2016, 137, 352-363.

[10] Burhan M, Chua KJE, Ng KC. Long term hydrogen production potential of concentrated photovoltaic (CPV) system in tropical weather of Singapore. International Journal of Hydrogen Energy 2016, 41(38), 16729-16742.

[11] Burhan M, Shahzad MW, Choon NK. Hydrogen at the Rooftop: Compact CPV-Hydrogen system to Convert Sunlight to Hydrogen. Applied Thermal Engineering 2018, 132, 154-164.

[12] Burhan M, Oh SJ, Chua KJ, Ng KC. Solar to hydrogen: Compact and cost effective CPV field for rooftop operation and hydrogen production. Applied Energy 2016 (article in press). http://dx.doi.org/10.1016/j.apenergy.2016.11.062

[13] Arndt R, Puto IR. Basic Understanding of IEC Standard Testing for Photovoltaic Panels. TUV SUD America Inc. http://www.tuvamerica.com/services/photovoltaics/articlebasicunderstandingpv.pdf. Date Retrieved: 21-10-2015.

[14] Burhan M, Shahzad MW, Ng KC. Sustainable Cooling with Hybrid Concentrated Photovoltaic Thermal (CPVT) System and Hydrogen Energy Storage. International Journal of Computational Physics Series 2018, 1(2), 40-51.

[15] Burhan M, Shahzad MW, Oh SJ, Ng KC. A pathway for sustainable conversion of sunlight to hydrogen using proposed compact CPV system. Energy Conversion and Management 2018, 165, 102-12.

[16] Muhammad B, Seung JO, Ng KC, Chun W. Experimental Investigation of Multijunction Solar Cell Using Two Axis Solar Tracker. In Applied Mechanics and Materials 2016 (Vol. 819, pp. 536-540). Trans Tech Publications.

[17] Oh SJ, Burhan M, Ng KC, Kim Y, Chun W. Development and performance analysis of a two-axis solar tracker for concentrated photovoltaics. International Journal of Energy Research 2015, 39(7), 965-76.

[18] Astronomical Applications Department of the U.S. Naval Observatory. http://aa.usno.navy.mil/data/docs/AltAz.php. Date Retrieved: 10-05-2015.

[19] Farahani S. ZigBee wireless networks and transceivers. newnes, 2011.

International Energy Agency, $\mathrm{CO}_{2}$ Emissions from Fuel Combustion Highlights, 2013.

[20] Burhan M. (2015). Theoretical and Experimental Study of Concentrated Photovoltaic (CPV) System with Hydrogen Production as Energy Storage (Doctoral dissertation).

[21] Lin-Heng L, Lok-Ming C, Chia A, Gunawansa A, Harn-Wei K. Sustainability Matters, Challenges and Opportunities in Environmental Management in Asia. Pearson Education South Asia Pte Ltd, 2011. Singapore. 